\title{
COMBUSTIÓN IN SITU HÚMEDA: ALTERNATIVA PARA EL RECOBRO MEJORADO EN COLOMBIA
}

\author{
Emilio A. Cavanzo ${ }^{1 *}$, Samuel F. Muñoz ${ }^{2}$, Hernando Bottía R. ${ }^{3}$, Eider Niz V. ${ }^{4}$,
} Aníbal Ordoñez R. ${ }^{5}$

*A quien debe dirigirse la correspondencia

\section{RESUMEN}

Durante la aplicación de la técnica de recobro mejorado del petróleo denominada combustión in situ, gran parte del calor generado durante la combustión se almacena en la arena calcinada detrás del frente de combustión y no se utiliza para el desplazamiento del aceite. La capacidad calorífica del aire seco es baja y el aire inyectado no puede transferir calor desde la matriz de la arena tan rápidamente como se genera. El agua por otra parte puede absorber y transportar el calor mucho más eficientemente que el aire. Si el aire se inyecta junto con el agua (combustión húmeda), el calor almacenado puede ser recuperado y transportado hacia adelante.

Para el presente estudio se desarrolló el modelo base de simulación numérica de la prueba de tubo de combustión húmeda realizada a un crudo colombiano, el simulador STARS de la compañía CMG. Para el desarrollo del mismo, se planteó un modelo cinético que describe el comportamiento oxidativo del crudo de estudio. Posteriormente, se realizó el ajuste histórico del modelo de simulación y se compararon los resultados numéricos con los resultados del modelo físico. Finalizado el modelo de simulación, se procedió a evaluar el efecto del agua sobre los parámetros de desempeño del modelo de la prueba de tubo de combustión húmeda, para lo cual se realizó un análisis de sensibilidad a la tasa de inyección de agua y se analizó su impacto sobre cada uno de los parámetros de desempeño.

Palabras clave: recobro mejorado del petróleo, combustión in situ, modelo de simulación, prueba de tubo de combustión, parámetros de desempeño.

\section{WET IN SITU COMBUSTION: ALTERNATIVE FOR ENHANCED OIL RECOVERY IN COLOMBIA}

\begin{abstract}
During the application of the enhanced oil recovery technique called in situ combustion, a great amount of the heat generated during combustion is stored in the burned sand behind the combustion front and is not used for oil displacement. The air heat capacity is low and the injected air can't transfer heat from the sand matrix as rapidly as is generated. Water, on the other hand, can absorb and transport heat much more efficiently than air. If air is injected with water, the heat stored sand can be recovered and transported forward.

For the present research it was developed the numerical simulation model of the wet combustion tube test made to Colombian crude by the STARS simulator of the CMG company. For the development of the model, it was proposed a kinetic model that describes the oxidative behavior of the studied crude. Subsequently, it was made the history matching of the simulation model and were compared the numerical results with the results of the physical model. Finalized the simulation model, it was evaluated the effect of water on the performance parameters of the tube test model of wet combustion, for which it was made a sensitivity analysis to the water injection rate and it was analyzed its impact on each performance parameter.
\end{abstract}

Keywords: enhanced oil recovery, in situ combustion, numerical simulation model, combustion tube test, performance parameters.

1. Universidad Industrial de Santander, UIS, Bucaramanga, Colombia. ecavanba@uis.edu.co

2. Universidad Industrial de Santander, UIS, Bucaramanga, Colombia. samuel@uis.edu.co

3. Instituto Colombiano del Petróleo, ECOPETROL S.A, Piedecuesta, Colombia. hernando.bottia@ecopetrol.com.co

4. Instituto Colombiano del Petróleo, ECOPETROL S.A, Piedecuesta, Colombia. eider.niz@ecopetrol.com.co

5. Instituto Colombiano del Petróleo, ECOPETROL S.A, Piedecuesta, Colombia. anibal.ordoñez@ecopetrol.com.co 


\section{INTRODUCCIÓN}

Una de las técnicas más antiguas y eficientes en el recobro de crudo pesado es la combustión in situ (CIS). A diferencia de una inyección convencional de gas, en un proceso CIS, el calor es usado como auxiliar para mejorar el recobro del aceite. El calor es generado en el yacimiento (in-situ) a partir de la quema de un material residual sólido de baja volatilidad, generado a partir del craqueo térmico del aceite, denominado coque [1]. El aceite es desplazado al pozo productor gracias al empuje de los gases de combustión y el agua producto de la combustión y la recondensación del agua de formación. En la figura 1 se pueden ver las zonas presentes en el yacimiento durante la aplicación de la técnica.

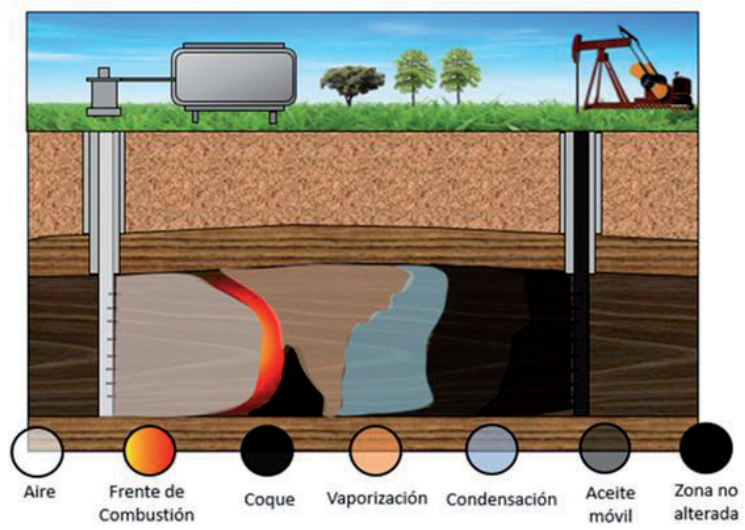

Figura 1. Zonas presentes en el yacimiento durante un proceso de combustión in situ.

A pesar de su alta eficiencia térmica, esta técnica no ha tenido gran auge debido al excesivo número de fallas del proceso, principalmente al ineficiente control del mismo y al desconocimiento de los fenómenos que ocurren durante la aplicación de la técnica, a tal punto que en Colombia únicamente se ha realizado una prueba piloto a nivel de campo [2].

Las características de depositación de coque en un yacimiento son los parámetros básicos para el diseño en la aplicación del proceso de combustión in situ. El coque es el combustible que sostiene el frente de combustión que resulta del craqueo térmico y la destilación del crudo próximo a la zona de combustión [3]. La cantidad de coque depositado es función de la gravedad API, saturación y movilidad del crudo, la presión y mineralogía del yacimiento, siendo mayor en crudos pesados, poco móviles, a alta presión. La depositación de coque determina la cantidad de aire que se requiere para el avance del frente de combustión, si el coque depositado es excesivo, lo cual es frecuente en el caso de los crudos pesados, se necesita una gran cantidad de aire para quemarlo en su totalidad reduciendo la viabilidad económica del proceso y limitando la aplicabilidad del mismo en yacimientos de crudo pesado [4].

Una estrategia para superar estas desventajas es por medio del proceso de combustión in situ húmeda, en el cual se inyecta agua simultánea o alternadamente con el aire. En el proceso de combustión in situ frontal seco, gran parte del calor generado durante la combustión se almacena en la arena calcinada detrás del frente y no se utiliza para el desplazamiento del aceite [5]. La capacidad calorífica del aire seco es baja y el aire inyectado no puede transferir calor desde la matriz de la arena tan rápidamente como se genera.

El agua por otra parte puede absorber y transportar el calor mucho más eficientemente que lo que puede el aire. Si el aire se inyecta junto con el agua, el calor almacenado en la arena quemada puede ser recuperado y transportado hacia adelante [6]. La combustión húmeda surge como un posible método de recobro para aumentar la productividad de los yacimientos colombianos.

En este proceso, el mecanismo de formación de coque, y por tanto el requerimiento de aire se reducen por medio de la mejora de la movilidad del crudo a través del calor transferido por el vapor y la interacción química entre el vapor y el crudo [7]. Esto reduce la cantidad de coque y por lo tanto el consumo de oxígeno necesario para su combustión.

Una de las pruebas más utilizadas para evaluar la viabilidad técnico - económica de implementar un proceso de combustión in situ, es la prueba de tubo de combustión. A partir de los resultados de la prueba experimental, se determinan los parámetros de desempeño del ensayo, como por ejemplo el requerimiento de aire y de combustible, los cuales son fundamentales para evaluar el éxito de la aplicación de un proyecto a escala piloto o escala de campo.

\section{ANTECEDENTES PRUEBAS DE TUBO DE COMBUSTIÓN HÚMEDA}

En la última década se han realizada una serie de investigaciones en relación a la combustión húmeda mediante pruebas de tubo, a continuación, se enuncian las más relevantes: Kok et al [8], realiza un estudio completo a través de pruebas de tubo, analizando principalmente el efecto del agua a RAA bajos y altos, en ciertos parámetros de desempeño del proceso de la combustión para crudos de diferente gravedad API. El 
requerimiento de aire y el consumo de combustible disminuyen con un aumento en la RAA, mientras que la relación aire - aceite inicialmente disminuye, pero tiende a un punto crítico a medida que continúa aumentando el RAA.

A partir de pruebas cinéticas, Castanier et al [9], concluyen que: el agua actúa en las reacciones LTO, reduciendo drásticamente el consumo de oxígeno. Esta reducción tiene un efecto indirecto en las reacciones HTO, aumentando el rango de temperatura durante el cual el oxígeno es consumido. Se evidenció un aumento en la producción de $\mathrm{CO}_{2}$ y una disminución en la producción de $\mathrm{CO}$, causada por la combustión del coque. El mejoramiento del crudo durante la combustión húmeda puede explicarse por el debilitamiento del régimen LTO causado por el vapor.

Debenest et al [10], realizan dos pruebas de tubo secas y tres pruebas húmedas. En las corridas de tubo húmedas, se observó un aumento en la velocidad del frente de combustión y del recobro del aceite, además de un exceso en la producción de $\mathrm{CO}_{2}$. Los autores recomiendan que el agua debe ser incluida en el mecanismo de reacción durante el modelamiento porque puede afectar en gran medida la combustión e indirectamente otros mecanismos como el transporte.

\section{METODOLOGÍA}

Para la presente investigación, se cuenta con los resultados de una prueba de tubo de combustión húmeda realizada a un crudo colombiano. La metodología de desarrollo es la siguiente:

- Desarrollo del modelo base de simulación numérica de la prueba de tubo mediante el simulador STARS de $\mathrm{CMG}$.

- Planteamiento de un modelo de reacciones químicas que describe el comportamiento oxidativo del crudo de estudio, a partir de los resultados experimentales y el balance másico de la prueba.

- Ajuste histórico del modelo de simulación, tomando los perfiles de temperatura como función objetivo y los parámetros cinéticos como parámetros de ajuste, mediante un procedimiento de ajuste manual.

- Comparación de los resultados numéricos con los resultados del modelo físico y cálculo del error relativo.

- Evaluación del efecto del agua sobre los parámetros de desempeño del modelo de la prueba de tubo de combustión húmeda, para lo cual se realizó un análisis de sensibilidad a la tasa de inyección de agua y se analizó su impacto sobre cada uno de los parámetros de desempeño, además se estudió su efecto sobre los perfiles de temperatura y la producción acumulada de aceite.

\section{PROCEDIMIENTO EXPERIMENTAL}

Un ensayo de tubo de combustión se puede separar en seis (6) pasos secuenciales:

- Caracterización y preparación de la roca y fluidos.

- Preparación de las pre-mezclas.

- Empaquetamiento y montaje del reactor.

- Verificación y alistamiento del equipo y sus periféricos.

- Inicio de la combustión.

- Cálculo y análisis de resultados.

El sistema de inyección de gases está encargado de suministrar el nitrógeno para la presurización del sistema y de aire para dar inicio a las reacciones de oxidación del sistema roca-fluido. El sistema de inyección de líquidos tiene la función de suministrar el crudo para saturar el medio poroso y agua para la combustión húmeda. El tubo de combustión (reactor) es donde ocurren todas las reacciones de oxidación, permite trabajar a una temperatura máxima de $815^{\circ} \mathrm{C}$ y una presión máxima de 4500 psi. El sistema de separación de fluidos como su nombre lo indica se encarga de separar la corriente líquida de la gaseosa.

El sistema de recolección de líquidos se encarga de recolectar y cuantificar el crudo y agua producido durante el ensayo. El sistema de recolección de gases tiene como función caracterizar los gases efluentes del ensayo. El sistema de purificación de gases tiene como función remover el ácido sulfúrico $\left(\mathrm{H}_{2} \mathrm{~S}\right)$ y el monóxido de carbono (CO) de la corriente gaseosa y el sistema de venteo está encargado de concentrar todos los gases efluente para emitirlos a la atmosfera.

\section{MODELO DE SIMULACIÓN NUMÉRICA}

Para el desarrollo del modelo base de simulación numérica, inicialmente se deben ingresar las propiedades de cada una de las siguientes secciones:

- Grid de simulación.

- Modelo de fluidos.

- Modelo cinético.

- Interacción roca - fluido.

- Condiciones iniciales.

- Pozos y eventos. 
A continuación, se describirán cada una de estas secciones y las propiedades asociadas a cada una de ellas.

Grid de simulación: El grid de simulación hace referencia al dimensionamiento y discretización a nivel espacial del modelo de simulación y la definición de las coordenadas a implementar. Además, es en esta sección donde se ingresan las propiedades petrofísicas (porosidad y permeabilidad) y las propiedades térmicas del sistema roca - fluido y el tubo de combustión. Teniendo en cuenta la configuración del tubo de combustión, se definió utilizar coordenadas radiales. En la tabla 1 se puede observar la discretización realizada al modelo de simulación.

Tabla 1. Propiedades del grid de simulación.

\begin{tabular}{cc} 
Parámetro & Valor \\
\hline Número de divisiones en $\mathrm{r}$ & 2 \\
\hline Número de divisiones en teta & 1 \\
\hline Número de divisiones en $\mathrm{k}$ & 128 \\
\hline Total de celdas & 256 \\
\hline
\end{tabular}

Las propiedades de porosidad y permeabilidad de la muestra se pueden ver en la tabla 2 junto con las dimensiones del tubo de combustión. La porosidad se determinó a partir de un balance volumétrico durante el empaquetamiento de la muestra y la permeabilidad a partir de la prueba de conectividad con nitrógeno. En la figura 2 se puede observar la discretización del tubo desde una vista frontal para la propiedad de temperatura y la ubicación de los calentadores.

Tabla 2. Dimensiones del tubo de combustión y propiedades petrofísicas de la muestra.

\begin{tabular}{|ccc|}
\hline Parámetro & Valor & Unidad \\
\hline Radio externo & 5,2303 & $\mathrm{~cm}$ \\
\hline Radio interno & 5,0293 & $\mathrm{~cm}$ \\
\hline Espesor pared tubo & 0,203 & $\mathrm{~cm}$ \\
\hline Espesor celda $\mathrm{k}$ & 1,309 & $\mathrm{~cm}$ \\
\hline Longitud del tubo & 201 & $\mathrm{~cm}$ \\
\hline Permeabilidad i, j, $\mathrm{k}$ & 5000 & $\mathrm{mD}$ \\
\hline Porosidad muestra & 0,336 & Frac \\
\hline Porosidad acero & 0 & Frac \\
\hline
\end{tabular}

Modelo de fluidos: Para el desarrollo del modelo de fluidos se cuenta con la composición del crudo colombiano. La composición es de interés para la agrupación del crudo por pseudocomponentes y el posterior desarrollo del modelo de reacciones.

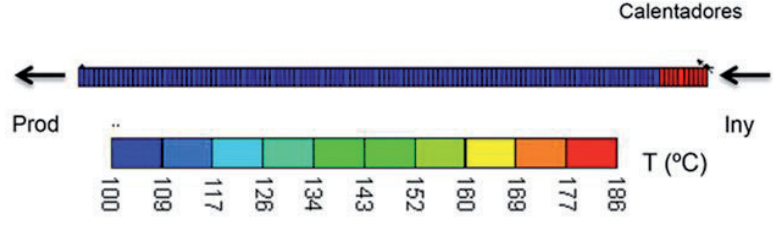

Figura 2. Discretización grid de simulación a nivel k.

El análisis cromatográfico realizado a la muestra de crudo colombiano presenta componentes lineales de 35 carbonos y sus isómeros, con una fracción pesada de $\mathrm{C}_{36+}$. Está es una distribución bastante extensa, típica de un crudo pesado de $9^{\circ} \mathrm{API}$. Algunas de las propiedades de interés del crudo se pueden ver en la tabla 3 , de las cuales está una gravedad API de $9^{\circ}$ y una densidad de $9 \mathrm{~g} / \mathrm{cc}$.

Tabla 3. Propiedades del crudo colombiano.

\begin{tabular}{|ccc|}
\hline Propiedad & Valor & Unidad \\
\hline Densidad crudo & 9 & ${ }^{\circ} \mathrm{API}$ \\
\hline Gravedad API crudo & 1,006 & $\mathrm{~g} / \mathrm{cc}$ \\
\hline Presión referencia & 3800 & $\mathrm{psi}$ \\
\hline Temperatura referencia & 85 & ${ }^{\circ} \mathrm{C}$ \\
\hline Presión superficie & 14,7 & $\mathrm{psi}$ \\
\hline Temperatura superficie & 25 & ${ }^{\circ} \mathrm{C}$ \\
\hline Peso molecular coque & 14,992 & $\mathrm{~g} / \mathrm{mol}$ \\
\hline Entalpía del coque & 17 & $\mathrm{~J} / \mathrm{gmol}-{ }^{\circ} \mathrm{C}$ \\
\hline
\end{tabular}

La primera agrupación por pseudocomponentes de un crudo fue propuesta por Adegbasan [11], para el modelamiento químico del aceite durante un proceso de combustión in situ, este modelo agrupa el crudo en una fracción liviana (maltenos), una fracción pesada (asfaltenos) y una fracción sólida (coque). Esta agrupación sería implementada posteriormente por Belgrave et al [12], para el crudo de Athabasca, obteniendo resultados satisfactorios al ajustar pruebas cinéticas, incluyendo el componente gas. Para la presente investigación se implementará esta agrupación, en la tabla 4 se pueden observar los pseudocomponentes del crudo colombiano, el porcentaje molar y el porcentaje en peso.

Tabla 4. Agrupación por pseudocomponentes del crudo colombiano.

\begin{tabular}{cccc} 
Pseudocomponente & $\mathbf{P M}(\mathbf{g} / \mathbf{m o l})$ & \% molar & \% peso \\
$\mathrm{CO}_{2}$ & 44,01 & 6,26 & 0,68 \\
$\mathrm{~N}_{2}$ & 28,0134 & 0,99 & 0,07 \\
\hline Gas $\left(\mathrm{C}_{1}-\mathrm{nC}_{4}\right)$ & 3,595 & 15,4 & 0,88 \\
\hline Maltenos $\left(\mathrm{C}_{5}-\mathrm{C}_{20}\right)$ & 58,023 & 28,8 & 14,02 \\
\hline Asfaltenos $\left(\mathrm{C}_{21}-\mathrm{C}_{36+}\right)$ & 341,752 & 48,55 & 84,35 \\
\hline Coque & 14,992 & - & - \\
\hline
\end{tabular}


En la figura 3 se puede observar el comportamiento de la viscosidad del aceite en función de la temperatura.

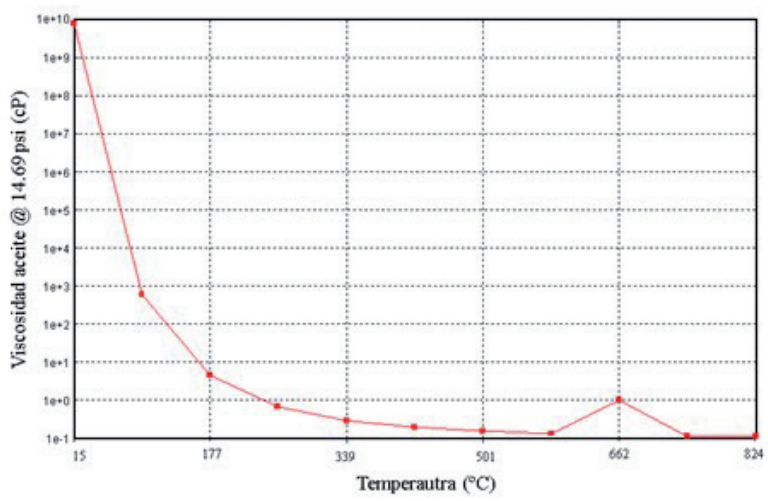

Figura 3. Variación de la viscosidad del aceite en función de la temperatura.

Modelo cinético: El modelo cinético para el crudo colombiano se planteó de acuerdo a los resultados experimentales de la prueba de tubo de combustión y una revisión de los principales modelos planteados en la literatura para los regímenes de reacción a baja, media y alta temperatura.

De acuerdo a la composición de los gases producidos de la prueba experimental, se identificaron los tres regímenes de reacción antes mencionados. Los coeficientes estequiométricos de las reacciones se plantearon a partir de la relación de pesos moleculares aparentes de los compuestos involucrados en la prueba y balances elementales. A continuación se muestran las reacciones planteadas para cada uno de los regímenes de reacción.

Reacciones de oxidación a baja temperatura (LTO): son reacciones exotérmicas, de adición de oxígeno. El mecanismo de reacción fue planteado por Burguer y Sahuquet [13]. Inicialmente los maltenos se oxidan parcialmente para producir asfaltenos, en la segunda reacción los asfaltenos se oxidan para producir coque (compuesto sólido de alto peso molecular). Las reacciones ocurren a bajas temperaturas y a partir éstas se obtiene la energía necesaria para generar la ignición. Se caracterizan por la adición de átomos de oxígeno a la cadena de hidrocarburos. Las reacciones LTO son las siguientes:

$$
\begin{aligned}
& \text { Maltenos }+1.62 \mathrm{O}_{2} \rightarrow 0.32 \text { Asfaltenos } \\
& \text { Asfaltenos }+28.630_{2} \rightarrow 83.92 \text { Coque }
\end{aligned}
$$

Modelo de reacciones a temperatura intermedia (ITO): En este modelo se plantea una reacción, en la cual la fracción pesada del crudo (asfaltenos) presenta craqueo térmico, pasando por un proceso consecutivo de deshidrogenación, craqueo y condensación.

El mecanismo de las reacciones a baja temperatura fue propuesto por Hayashitani [14]. En la etapa de deshidrogenación, los átomos de hidrógeno son arrancados de las moléculas, mientras que los átomos de carbono no son alterados.

Durante el craqueo, se rompen los enlances $\mathrm{C}-\mathrm{C}$ y se forman las moléculas más pequeñas. Finalmente, en la etapa de condensación el número de carbonos en la molécula aumenta y se forma un compuesto de alto peso molecular, denominado coque. A continuación se muestra la reacción planteada:

$$
\text { 1.9 Asfaltenos } \rightarrow 34.5 \text { Coque }+2.34 \text { Maltenos }
$$

Modelo de reacciones a alta temperatura (HTO): En el régimen HTO, los asfáltenos dominan la liberación de calor y el proceso en términos de flujo de calor. En una reacción exotérmica, cuando la energía liberada no es retirada del sistema, en este caso del yacimiento, la temperatura comenzará a incrementarse. Al aumentar la temperatura, la velocidad de reacción aumenta, con lo cual se libera más energía.

Estudios han mostrado que la propagación del frente de combustión es el método más rápido de recobro térmico. Ramey [15] mostró que la velocidad de la ola de calor de convección es cerca de un cuarto del frente de combustión. El mecanismo de la reacción HTO fue propuesto por Benham et al [16]. A continuación se muestra la reacción planteada:

$$
\text { Coque }+79.70_{2} \rightarrow \beta 52+8.24 \mathrm{CO}+2.53 \mathrm{H}_{2} \mathrm{O}
$$

Determinación de los parámetros cinéticos: La determinación de los parámetros cinéticos se debe realizar mediante pruebas cinéticas [17], como la prueba RTO (Ramped Temperature Oxidation), DTA (Differential Thermo Analysis) o la prueba TGA (Thermo Gravimetric Analysis). 
Para la presente investigación no se cuenta con este tipo de pruebas, no obstante numerosas investigaciones han demostrado que la cinética varía dependiendo de la escala en la cual se aplique la técnica, razón por la cual los resultados de una prueba cinética no serían aplicables al modelamiento cinético de una prueba de tubo de combustión. Teniendo en cuenta que las pruebas cinéticas se realizan en muestras de pocos centímetros de espesor y las pruebas de tubo se realizan en muestran de pies de espesor, para la prueba de tubo de combustión húmeda se determinaron los parámetros cinéticos (energía de activación y factor de frecuencia) y la entalpía de reacción (parámetro termodinámico) por un método de ajuste manual en el simulador numérico. Para el desarrollo de modelos de simulación de pruebas de tubo de combustión, autores como Kapadia et al [18], han determinado los parámetros de la ecuación de Arrhenius mediante un proceso de ajuste manual en el simulador numérico, obteniendo resultados satisfactorios.

Entonces, se tomaron como parámetros de ajuste los parámetros cinéticos, y como función objetivo los perfiles de temperatura de la prueba. Finalmente, los parámetros cinéticos que mejor se ajustaron a los resultados de la prueba experimental se muestran en la tabla 5.

Tabla 5. Parámetros cinéticos modelo de simulación numérica.

\begin{tabular}{cccc} 
Reacción & $\begin{array}{c}\text { Energía de } \\
\text { activación, (BTU/ } \\
\text { lbmol) }\end{array}$ & $\begin{array}{c}\text { Factor de } \\
\text { frecuencia }\end{array}$ & $\begin{array}{c}\text { Entalpía } \\
\text { (BTU / } \\
\mathbf{l b m o l )}\end{array}$ \\
\hline LTO -1 & $8 * 10^{3}$ & $1 * 10^{4}$ & $1.1 * 10^{7}$ \\
\hline LTO - 2 & $1 * 10^{4}$ & $1 * 10^{7}$ & $1.1 * 10^{7}$ \\
\hline ITO & $3.5 * 10^{4}$ & $1.5 * 10^{7}$ & 0 \\
\hline HTO & $1 * 10^{3}$ & $8 * 10^{6}$ & $1.8 * 10^{6}$ \\
\hline
\end{tabular}

Interacción roca - fluido: La interacción roca - fluido se representa mediante las curvas de permeabilidad relativa, las cuales describen el flujo del agua y el aceite en el medio poroso, despreciando los efectos capilares. Las curvas fueron generadas a partir de los end points (ver tabla 6) de la prueba experimental, los cuales se determinaron durante el empaquetamiento de la muestra y la prueba de conectividad, las curvas se calcularon a partir de la correlación de Stone [19], estas se pueden ver en la figura 4.

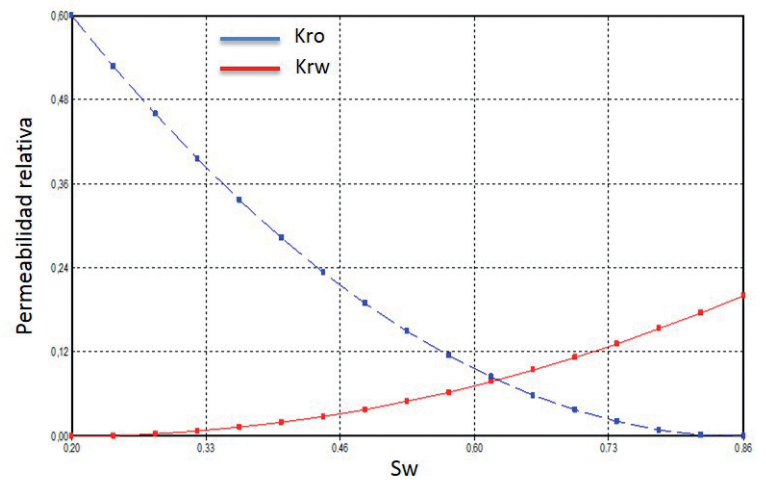

Figura 4. Curvas de permeabilidad relativa.

Tabla 6. End points curvas de permeabilidad relativa.

\begin{tabular}{|cc|}
\hline End point & Valor \\
\hline Saturación de agua connata & 0,14 \\
\hline Saturación de agua crítica & 0,14 \\
\hline Saturación de aceite irreducible (Curva agua - aceite) & 0,2 \\
\hline Saturación de aceite residual (Curva agua - aceite) & 0,2 \\
\hline Saturación de aceite irreducible (Curva gas - líquido) & 0,15 \\
\hline Saturación de aceite residual (Curva gas - líquido) & 0,15 \\
\hline $\begin{array}{c}\text { Saturación de gas connata } \\
\text { Saturación crítica de gas }\end{array}$ & 0,05 \\
\hline $\begin{array}{c}\text { Permeabilidad relativa al aceite @ saturación de agua } \\
\text { connata }\end{array}$ & 0,05 \\
\hline Permeabilidad relativa al agua @ saturación de aceite & 0,6 \\
\hline irreducible & 0,2 \\
\hline Permeabilidad relativa al gas @ saturación de líquido \\
connata
\end{tabular}

Condiciones iniciales: Esta sección hace referencia a las condiciones iniciales en las cuales se encuentra el modelo de simulación, para lo cual se cuenta con las saturaciones iniciales de fluidos, presión y temperatura inicial, y por último la composición del aceite para cada uno de los pseudocomponentes (ver tabla 7). 
Tabla 7. Condiciones iniciales modelo de simulación.

\begin{tabular}{ccc}
\hline Parámetro & Valor & Unidad \\
\hline Presión inicial & 3800 & $\mathrm{psi}$ \\
\hline Temperatura inicial & 85 & ${ }^{\circ} \mathrm{C}$ \\
\hline So & 0,5674 & - \\
\hline $\mathrm{Sw}$ & 0,1345 & - \\
\hline $\mathrm{Sg}$ & 0,2981 & - \\
\hline Fracción molar $\mathrm{CO}_{2}$ & 0,0626 & - \\
\hline Fracción molar $\mathrm{N}_{2}$ & 0,0099 & - \\
\hline Fracción molar Gas & 0,154 & - \\
\hline Fracción molar Maltenos & 0,288 & - \\
\hline Fracción molar Asfaltenos & 0,4855 & - \\
\hline
\end{tabular}

Pozos y eventos: Inicialmente se realizó una prueba de conectividad a la muestra, inyectando nitrógeno durante 12 horas. Posteriormente se dio inicio a la inyección de aire durante 1,93 horas, en esta etapa se produjo la ignición. Finalizada la inyección de aire, se inició la combustión húmeda, la cual tuvo una duración de 9,83 horas. El calentador se ubicó en las primeras 10 celdas en dirección $\mathrm{K}$ del modelo de simulación.

La temperatura inicial de los calentadores es de $85^{\circ} \mathrm{C}$ y se incrementó a $320^{\circ} \mathrm{C}$ al iniciar la inyección de aire, teniendo en cuenta que a esta temperatura se alcanzó la ignición en el modelo físico, hasta el final de la prueba. Se implementó un delta de tiempo de 0,5 minutos. En la tabla 8 se pueden observar los parámetros operacionales para cada uno de los eventos del pozo inyector y del pozo productor.

Tabla 8. Parámetros operacionales pozos inyector y productor.

\begin{tabular}{|c|c|c|}
\hline \multicolumn{3}{|c|}{ Inyección de nitrógeno } \\
\hline Fecha inicio & 0 & $\min$ \\
\hline Fecha fin & 720 & $\min$ \\
\hline Presión de fondo fluyente (BHP) & 3800 & psi \\
\hline Caudal de inyección & 5000 & $\mathrm{~cm}^{3} / \mathrm{min}$ \\
\hline Temperatura de inyección & 85 & ${ }^{\circ} \mathrm{C}$ \\
\hline \multicolumn{3}{|c|}{ Inyección de aire } \\
\hline Fecha inicio & 721 & $\min$ \\
\hline Fecha fin & 834 & $\min$ \\
\hline Presión de fondo fluyente (BHP) & 3800 & PSI \\
\hline Caudal de inyección & 5000 & $\mathrm{~cm}^{3} / \min$ \\
\hline Temperatura de inyección & 85 & ${ }^{\circ} \mathrm{C}$ \\
\hline Fracción volumétrica $\mathrm{N}_{2}$ & 0,79 & - \\
\hline Fracción volumétrica $\mathrm{O}_{2}$ & 0,21 & - \\
\hline \multicolumn{3}{|c|}{ Inyección de aire + agua } \\
\hline Fecha inicio & 835 & $\min$ \\
\hline Fecha fin & 1425 & $\min$ \\
\hline Presión de fondo fluyente (BHP) & 3800 & psi \\
\hline Caudal de inyección aire & 5000 & $\mathrm{~cm}^{3} / \min$ \\
\hline Caudal de inyección agua & 8000 & $\mathrm{~cm}^{3} / \min$ \\
\hline Temperatura de inyección & 85 & ${ }^{\circ} \mathrm{C}$ \\
\hline \multicolumn{3}{|c|}{ Pozo productor } \\
\hline Fecha inicio & 0 & $\min$ \\
\hline Fecha fin & 1425 & $\min$ \\
\hline Presión de producción & 3220 & psi \\
\hline
\end{tabular}




\section{RESULTADOS}

Finalizado el desarrollo del modelo base de simulación numérica, se procedió a visualizar los resultados y realizar el ajuste histórico del modelo, teniendo en cuenta que el objetivo de la simulación numérica de yacimientos es reproducir el comportamiento del yacimiento, para este caso en particular el objetivo del modelo de simulación es reproducir la prueba de tubo de combustión.

Perfiles de temperatura: En las figuras 5, 6 y 7 se puede observar el ajuste de los perfiles de temperatura para las zonas $1-5,6-10$ y 11-15, respectivamente.

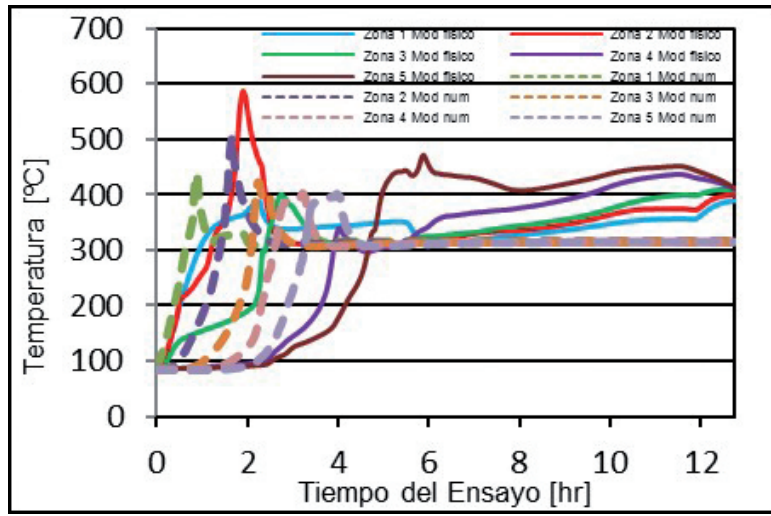

Figura 5. Ajuste perfiles de temperatura zonas 1-5.

La escala de tiempo de las gráficas corresponde al periodo de inyección de aire y la combustión húmeda únicamente, ya que el periodo de la inyección de nitrógeno tiene por objetivo simular la prueba de conectividad.

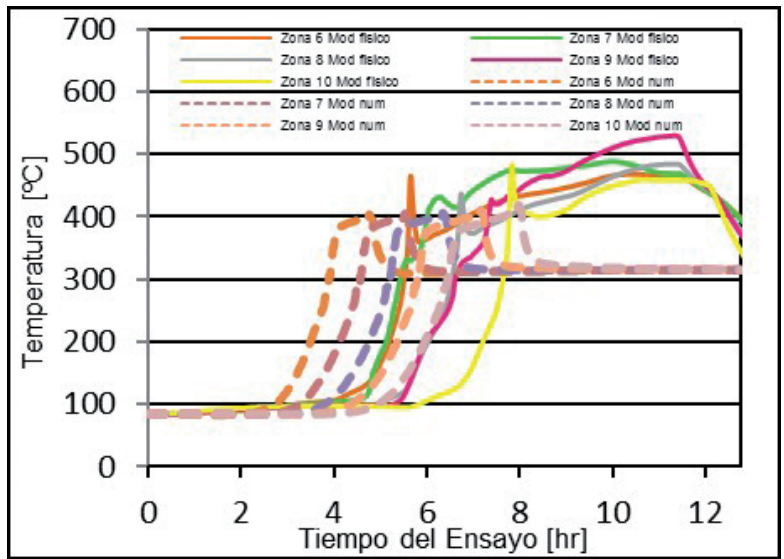

Figura 6. Ajuste perfiles de temperatura zonas 6-10.

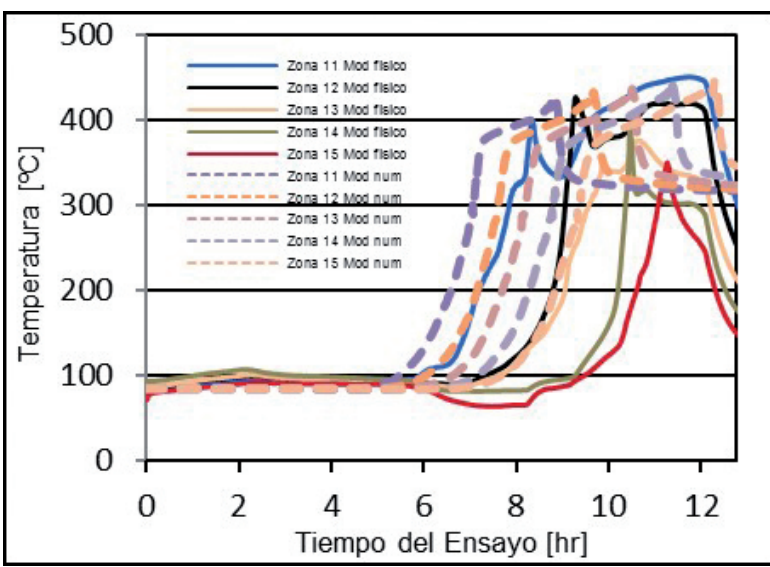

Figura 7. Ajuste perfiles de temperatura zonas 11-15.

Para lograr el ajuste se tomó como referencia la energía de activación, el factor de frecuencia y la entalpía de las reacciones de oxidación.

Composición de los gases producidos: En la figura 8 se puede ver el ajuste logrado de la composición de gases producidos. La producción de oxígeno en el modelo de simulación numérica es nula, por lo cual se puede afirmar que todo el oxígeno fue consumido en las reacciones de oxidación. A partir de la hora 0,5 la producción de $\mathrm{CO}_{2}$ se estabiliza aproximadamente en $10,1 \%$ y la producción de $\mathrm{CO}$ en $1,58 \%$.

El volumen de gases producidos del modelo de simulación depende fundamentalmente de la estequiometría del modelo de reacciones, la cual se planteó en base a los resultados experimentales y el balance másico de la prueba.

Las diferencias observadas en el volumen de gases producidos del modelo físico y el modelo numérico se deben principalmente a ciertos fenómenos involucrados durante la combustión, como la difusión y dispersión molecular, los cuales no son fielmente representados por el modelo numérico, además del orden de aproximación asociado al error de truncamiento implícito en el modelo numérico.

En el modelo físico, hay una producción de oxígeno en el periodo 3,5 - 7,5 horas, lo cual es característico de las reacciones de craqueo térmico. La producción de $\mathrm{CO}$ y $\mathrm{CO}_{2}$ inicia en el mismo momento en el cual comienza la inyección de agua (1,93 h), mientras que en el modelo numérico la producción de estos gases inicia a partir de 
la hora 0,1 . El inicio temprano de la producción de gases en el modelo numérico es congruente con la rápida ignición observada en el perfil de temperaturas, ya que el primer pico es monitoreado por la primera termocupla, mientras que en el modelo físico es monitoreado por la segunda termocupla.

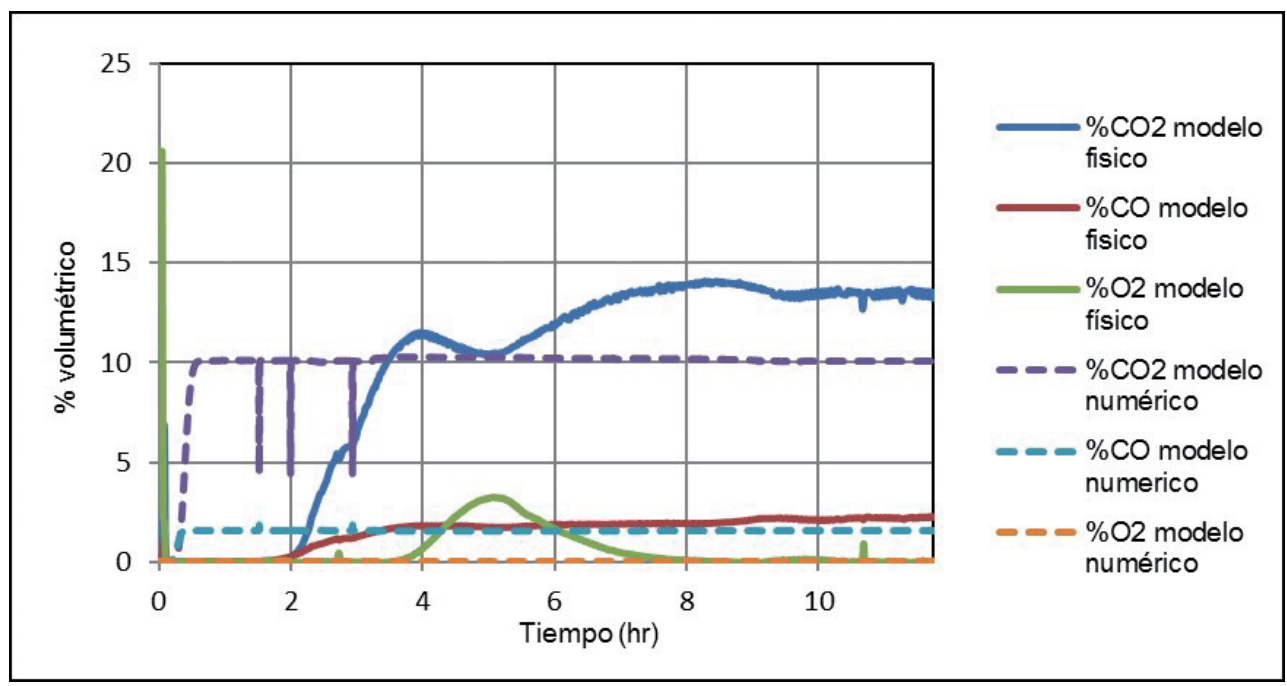

Figura 8. Ajuste histórico de la composición de los gases producidos.

Velocidad del frente de combustión: En la figura 9 se puede observar el ajuste de la velocidad del frente de combusitón. La velocidad del frente corresponde a la pendiente de la gráfica distancia del frente desde el inyector vs tiempo del ensayo a $350^{\circ} \mathrm{C}$ en el periodo de tiempo en el cual la combustión es estable. Se escogió esta temperatura durante el desarrollo del experimento ya que a $350^{\circ} \mathrm{C}$ predominan las reacciones HTO.

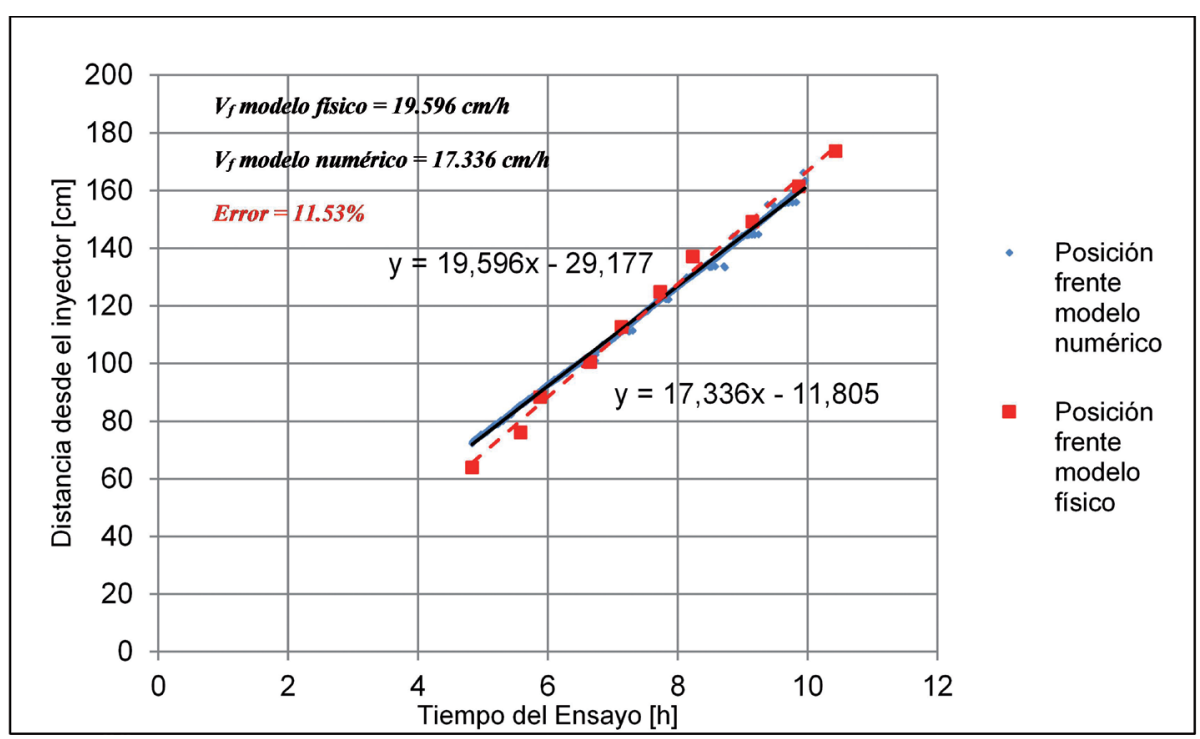

Figura 9. Ajuste histórico de la velocidad del frente de combustión. 
La velocidad del frente del modelo físico es de $19,596 \mathrm{~cm} / \mathrm{h}$, mientras que la velocidad del frente del modelo numérico es de $17,336 \mathrm{~cm} / \mathrm{h}$, obteniendo un error de $11,53 \%$. La gráfica se construyó en el periodo de tiempo 4,8 - 10 horas, ya que en este periodo el frente de combustión se mantuvo estable en su recorrido a través del tubo y es posible obtener un valor representativo de la velocidad del frente.
El ajuste obtenido es aceptable, teniendo en cuenta que la leve diferencia se debe al orden de aproximación inherente al error de truncamiento del modelo numérico, el cual se genera durante la discretización por diferencias finitas de la ecuación de difusividad que describe el flujo de fluidos en el medio poroso.

Producción acumulada de líquido: En la figura 10 se encuentra el ajuste de la producción acumulada de líquido.

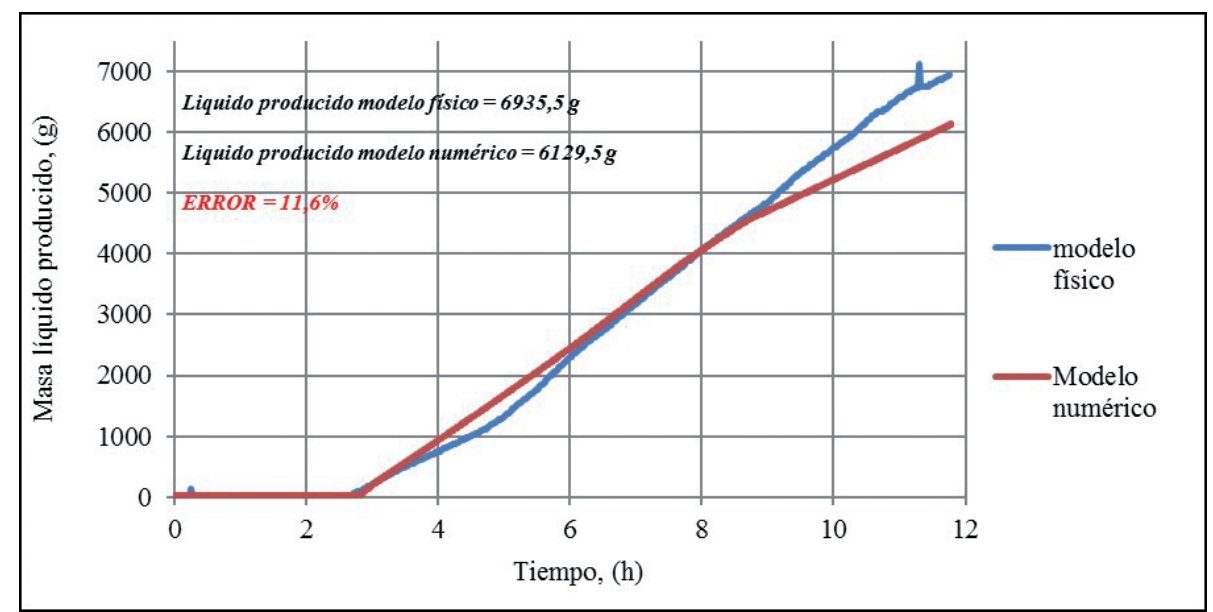

Figura 10. Ajuste histórico de la producción acumulada de líquido.

El líquido total producido del modelo físico es de 6935,5 g, mientras que el líquido producido del modelo numérico es de $6129,5 \mathrm{~g}$, obteniendo un error de 11,6\%. A partir de la hora 2,8 comienza la producción de líquido en ambos modelos.

En la hora 8,8 la tasa de aumento de líquido del modelo numérico comienza a decaer, mientras que la del modelo físico se mantiene estable, esta diferencia se puede explicar debido a que en la prueba experimental intervienen ciertos fenómenos y mecanismos de desplazamiento adicionales que contribuyen al recobro de líquido, y que no son fielmente representados por el modelo numérico, como son la difusión del oxígeno del gas de inyección en la interface del combustible y la desorción de los productos de la combustión.

\section{EFECTO DEL AGUA SOBRE LOS PARÁMETROS DE DESEMPEÑO}

Para analizar el efecto del agua sobre los parámetros de desempeño de la prueba de tubo, se tomó como referencia el modelo de simulación ajustado y se realizó un análisis de sensibilidad variando únicamente la tasa de inyección de agua. Finalmente, a partir de las variables de salida del modelo, se calcularon los parámetros de desempeño.

En la figura 11 se encuentra la velocidad del frente de combustión para diferentes tasas de agua., se puede observar que a una relación de inyección agua / aire de $0,384 \mathrm{~kg} / \mathrm{m}^{3}$, la velocidad del frente de combustión es máxima $(31,6 \mathrm{~cm} / \mathrm{h})$. 
Combustión in situ húmeda: alternativa para el recobro mejorado en Colombia

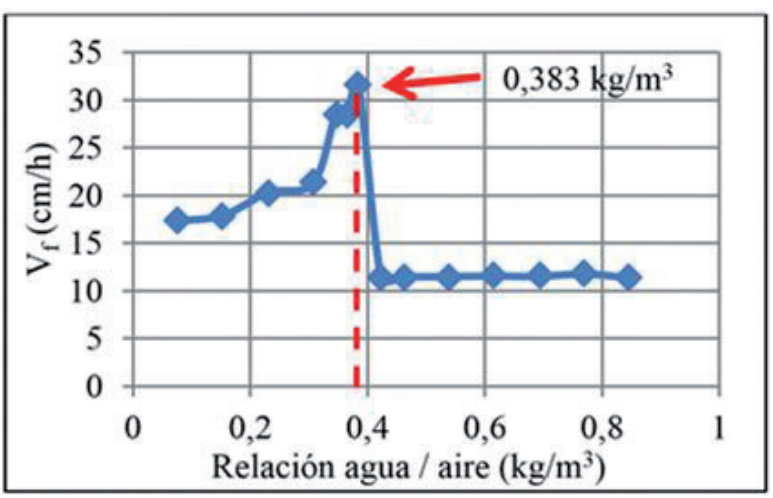

Figura 11. Efecto del agua sobre la velocidad del frente de combustión.

Lo anterior corrobora estudios como los de Bagci [21], en el cual afirma que a tasas altas de agua la velocidad del frente de combus tión aumenta debido al aumento del calor transferido por vapor delante del frente de combustión (transferencia de calor por convección), haciendo que el proceso sea más eficiente desde el punto de vista de transferencia de energía.

Sin embargo, a medida que la tasa de agua continúa aumentando, la velocidad del frente de combustión disminuye hasta estabilizarse en $11,5 \mathrm{~cm} / \mathrm{h}$. Smith et al [22] denominan este fenómeno como el efecto de enfriamiento, en el cual se alcanzan condiciones de combustión súper húmeda y el frente de combustión se apaga parcialmente, los autores afirman que el enfriamiento que genera el agua inyectada puede promover altos regímenes LTO y un régimen HTO muy tenue o nulo.

Entonces, a partir de una relación de inyección agua / aire (RAA) de $0,42 \mathrm{~kg} / \mathrm{m}^{3}$ comienza a predominar el efecto de enfriamiento y la condición de combustión súper húmeda, a RAA inferiores a esta predomina la combustión húmeda normal y/o completa.

Requerimiento de aire (RA): Es el volumen de aire requerido para quemar una unidad de volumen de yacimiento, se calcula de la siguiente forma:

$$
R A=\frac{F A}{V f}
$$

RA: Requerimiento de aire, $\left[\frac{\mathrm{m}^{3}(\mathrm{ST})}{\mathrm{m}^{3}}\right]$

FA: Flujo de aire, $\left[\frac{\mathrm{m}}{\mathrm{h}}\right]$
$\mathrm{V}_{\mathrm{f}}$ : Velocidad del frente de combustión, $\left[\frac{\mathrm{cm}}{\mathrm{h}}\right]$

En la figura 12 se puede ver la gráfica de requerimiento de aire en función de la relación agua / aire (RAA). Este es un parámetro que determina en gran medida la viabilidad económica de implementar un proyecto de combustión in situ, ya que a partir del volumen de aire requerido se dimensionan los compresores.

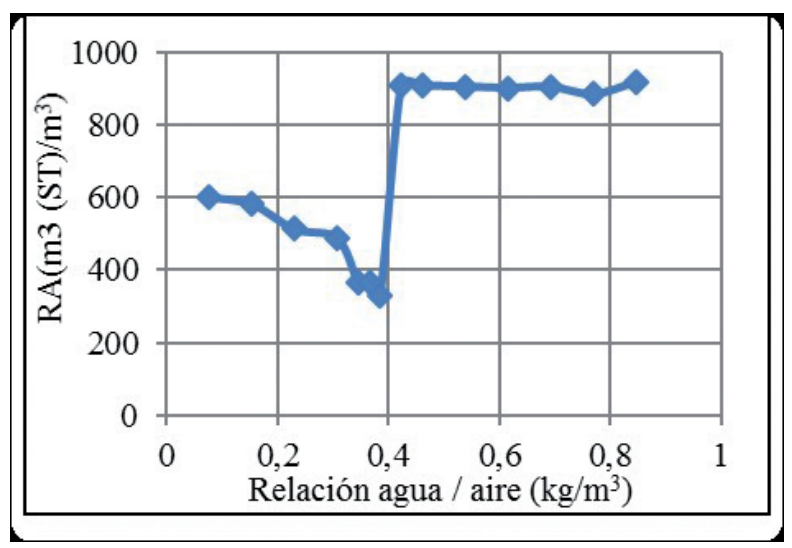

Figura 12. Efecto del agua sobre el requerimiento de aire.

A una RAA de $0,384 \mathrm{~kg} / \mathrm{m}^{3}$ el requerimiento de aire es mínimo $\left(330 \mathrm{~m}^{3}(\mathrm{ST}) / \mathrm{m}^{3}\right)$. Este efecto ha sido observado por autores como Garon y Moore [23], a partir de las pruebas de tubo desarrolladas por los autores, han concluido que el aire requerido es menor para la combustión húmeda en comparación a la seca, teniendo en cuenta que el combustible depositado bajo estas condiciones es menor. Así mismo, Dietz y Weijdema [24] determinaron que a condiciones de combustión húmeda completa (tasas de inyección de agua intermedias) el requerimiento de aire es mínimo.

A RAA superiores a $0,384 \mathrm{~kg} / \mathrm{m}^{3}$, el requerimiento de aire aumenta considerablemente hasta estabilizarse en $900 \mathrm{~m}^{3}(\mathrm{ST}) / \mathrm{m}^{3}$, esto se debe a que el frente de combustión bajo estas condiciones se ha apagado parcialmente, la temperatura disminuye hasta alcanzar el nivel de la meseta de vapor siendo predominante el frente de vaporización, por lo cual se requiere una gran cantidad de aire para quemar una unidad de roca.

Requerimiento de combustible (RC): Es teóricamente la masa de hidrocarburo que es consumido para el proceso de combustión.

$$
R C=\frac{C C}{V B R}
$$


$\mathrm{RC}$ : Requerimiento de combustible, $\left[\frac{\mathrm{kg}}{\mathrm{m}^{3}}\right]$

$\mathrm{CC}$ : Combustible consumido, $[\mathrm{kg}]$

VBR: Volumen barrido del reactor, $\left[\mathrm{m}^{3}\right]$

En la figura 13 se puede ver la gráfica de requerimiento de combustible en función de la RAA. Este parámetro es directamente proporcional al combustible consumido. De la figura se puede observar que el requerimiento de combustible se mantiene en un rango de $57-58,2 \mathrm{~kg} / \mathrm{m}^{3}$.

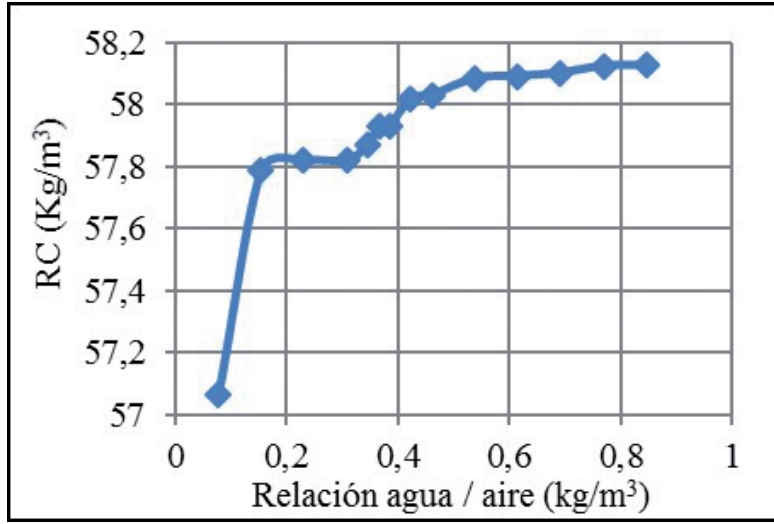

Figura 13. Efecto del agua sobre el requerimiento de combustible.

El leve aumento del requerimiento de combustible al incrementarse la RAA está ligado directamente al requerimiento de aire, ya que este parámetro a RAA superiores a $0,384 \mathrm{~kg} / \mathrm{m}^{3}$ aumenta hasta $900 \mathrm{~m}^{3}$ (ST) $/ \mathrm{m}^{3}$. A medida que aumenta el requerimiento de aire, también aumenta el requerimiento de combustible, pues es necesaria una mayor cantidad de aire para quemar un volumen de roca. Además, en condiciones de combustión súper húmeda, al ser la temperatura considerablemente menor que en la combustión húmeda normal, las reacciones LTO predominan debido al efecto de enfriamiento generado por las altas tasas de agua, por lo cual el volumen de hidrocarburo depositado es mayor en comparación al caso en el cual las temperaturas típicas de la combustión son predominantes (combustión seca o húmeda normal).

Relación aire / combustible: Es el volumen de aire requerido para quemar una unidad de masa del combustible, está en función de la cantidad de carbón e hidrógeno del combustible y la relación de nitrógenooxigeno del gas de inyección.

$$
R A C=\frac{R A}{R C}
$$

RAC: Relación aire / combustible, $\left[\frac{m^{3}(S T)}{k g}\right]$

RA: Requerimiento de aire, $\left[\frac{\mathrm{m}^{3}(\mathrm{ST})}{\mathrm{m}^{3}}\right]$

RC: Requerimiento de combustible, $\left[\frac{\mathrm{kg}}{\mathrm{m}^{3}}\right]$

En la figuras 14 se encuentra la gráfica de relación aire / combustible. La relación aire / combustible (RAC) es proporcional al requerimiento de aire, por lo cual mantienen la misma tendencia. Este es un parámetro fundamental a la hora de evaluar la viabilidad técnica económica de implementar un proyecto de combustión in situ a escala piloto o escala de campo, en la práctica son deseables valores de relación aire / combustible bajos, ya que esto marca un indicio de que el proceso se está efectuando de manera adecuada y se mantiene en régimen HTO, pues se requieren bajos volúmenes de aire para quemar una unidad másica de combustible.

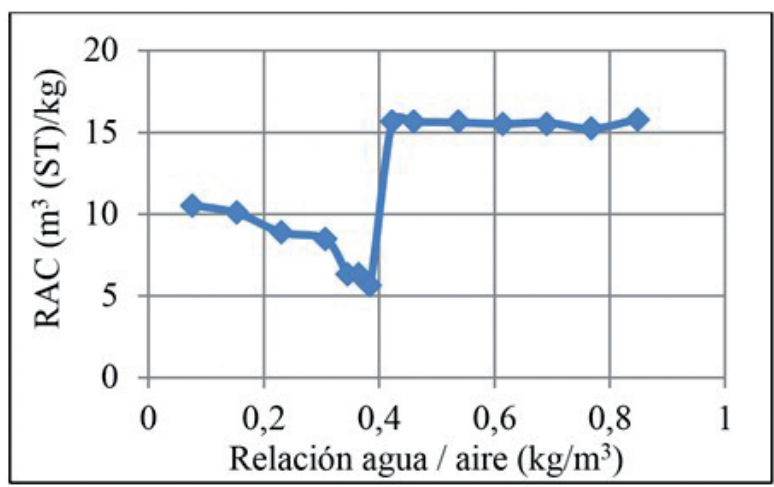

Figura 14. Efecto del agua sobre la relación aire / combustible.

A una RAA de $0,384 \mathrm{~kg} / \mathrm{m}^{3}$ la relación aire / combustible es mínima $\left(5,68 \mathrm{~m}^{3}(\mathrm{ST}) / \mathrm{kg}\right)$. A medida que aumenta la RAA, la RAC aumenta hasta estabilizarse en $15,5 \mathrm{~m}^{3}$ (ST) $/ \mathrm{kg}$. Entonces, a RAA entre $0,07-0,384 \mathrm{~kg} / \mathrm{m}^{3}$ se puede afirmar que la combustión se mantiene en régimen HTO y la combustión es húmeda normal, teniendo en cuenta los bajos valores de RAC y requerimiento de aire, y a RAA superiores a $0,384 \mathrm{~kg} / \mathrm{m}^{3}$ predomina el régimen LTO y la combustión es súper húmeda. 


\section{CONCLUSIONES}

- Los perfiles de temperatura del modelo numérico y el modelo físico mantienen el mismo rango de variación de la temperatura entre el plateau y el frente de combustión, el cual se encuentra entre 350 y $450{ }^{\circ} \mathrm{C}$. La meseta de vapor del modelo numérico se mantiene en $320^{\circ} \mathrm{C}$, mientras que el plateau del modelo físico tiende a aumentar a temperaturas superiores a $400^{\circ} \mathrm{C}$, este es un efecto típico del proceso de combustión húmeda, en el cual la meseta de vapor es considerablemente más amplia que la que caracteriza a aquella de la combustión seca.

- La producción de oxígeno del modelo numérico es nula, indicando que este se consume en su totalidad durante las reacciones de oxidación, a diferencia del modelo físico en el cual se obtuvo una producción de $25 \mathrm{~g}$. El inicio temprano de la producción de gases en el modelo numérico es congruente con la rápida ignición observada en el perfil de temperaturas.

- Para una relación de inyección agua / aire de 0.384 $\mathrm{kg} / \mathrm{m}^{3}$ la velocidad del frente de combustión es máxima $(0.316 \mathrm{~m} / \mathrm{h})$ y el requerimiento de aire es mínimo $\left(329.44 \mathrm{~m}^{3}(\mathrm{ST}) / \mathrm{m}^{3}\right)$, lo cual indica que esta es la tasa de inyección óptima para el modelo de simulación numérica de la prueba de tubo húmeda y la combustión es húmeda completa.

- A RAA superiores a $0,384 \mathrm{~kg} / \mathrm{m}^{3}$, el requerimiento de aire aumenta considerablemente hasta estabilizarse en $900 \mathrm{~m}^{3}(\mathrm{ST}) / \mathrm{m}^{3}$, esto se debe a que el frente de combustión bajo estas condiciones se ha apagado parcialmente, la temperatura disminuye hasta alcanzar el nivel de la meseta de vapor siendo predominante el frente de vaporización, por lo cual se requiere una gran cantidad de aire para quemar una unidad de roca.

- A relaciones de inyección agua / aire superiores a $0.42 \mathrm{~kg} / \mathrm{m}^{3}$ se observa que la relación aire / combustible y oxígeno combustible se estabiliza $15.5 \mathrm{~m}^{3}(\mathrm{ST}) / \mathrm{m}^{3}$ y $3.2 \mathrm{~m}^{3}(\mathrm{ST}) / \mathrm{m}^{3}$ respectivamente, los cuales son valores considerablemente altos para un proceso de combustión in situ, indicando que se requiere una gran cantidad de aire para quemar una unidad volumétrica de roca, estas son características de la combustión súper húmeda, mientras que a una RAA de $0,384 \mathrm{~kg} / \mathrm{m}^{3}$ estos parámetros alcanzan valores mínimos, indicando que a esta tasa de inyección predomina el régimen HTO.
- El combustible consumido se estabiliza en 0.83 $\mathrm{kg}$ y el requerimiento de combustible en $58 \mathrm{~kg} / \mathrm{m}^{3}$ a altas tasas de inyección de agua, los cuales son valores considerablemente altos, indicando que la combustión a estas tasas es súper húmeda.

- El presente estudio perfila a la combustión húmeda como una aplicación potencial más eficiente desde el punto de vista técnico - económico en relación a la combustión seca, esto debido al menor requerimiento de aire, lo cual reduce notablemente los costos de compresión del proceso.

\section{REFERENCIAS BIBLIOGRÁFICAS}

1. SARATHI. P.S. In Situ Combustion Handbook - Principles and Practices. National Petroleum Technology Office, Tulsa, Oklahoma. 1998.

2. G. MAYA, D. MERCADO, R. CASTRO, M. TRUJILLO, C. SOTO. Enhanced Oil Recovery (EOR) Status- Colombia. SPE Latin American and Caribbean Petroleum Engineering Conference. Lima, Perú. SPE-139199-MS. 2010.

3. A.LAPENE, L.M. CASTANIER, G.DEBENEST, M. QUINTARD, A.M. KAMP, B. CORRE. Effects of Steam on Heavy Oil Combustion. SPE 118800. 2009.

4. FASSIHI M.R. Analysis of fuel oxidation in in situ combustion oil recovery. $\mathrm{Ph}$. D. Thesis, Stanford University. 1981.

5. GREAVES M, FIELD R.W, ADEWUSI V.A. In situ combustion for oil recovery. Chem. Eng. Res. Design 65:23-28. 1988.

6. GREEN. D.W, WILLHITE, G.P. Enhanced Oil Recovery. 1998. Pg 376.

7. URSENBACH M.G. An overview of laboratory in situ combustion Behavior of Athabasca oil sands. MSc. Thesis. University of Calgary. 1998.

8. BAGCI S, KOK M.B. In Situ Combustion Laboratory Studies of Turkish Heavy Oil Reservoirs. ELSEVIER, Department of Petroleum and Natural Gas Engineering: Middle East Technical University, Turkey. 2001. 
9. LAPENE A, CASTANIER L.M, DEBENEST G, QUINTARD A, KAMP A.M, CORRE B. Effects of Steam on Heavy Oil Combustion. SPE 118800. 2009.

10. LAPENE A, CASTANIER L.M, DEBENEST G, QUINTARD A, KAMP A.M, CORRE B. Effects of Water on Kinetics on In-Situ Combustion. SPE 121180. 2009.

11. ADEGBASAN, DONELLY, R.G MOORE. Liquid phase oxidation kinetics of oil sands bitumen: Models for in situ combustion numerical simulators. SPE-12004-PA. 1987.

12. BELGRAVE J.D.M, MOORE R.G, URSENBACH M.G, BENNION D.W. A comprehensive approach to in situ combustion modeling. University of Calgary. SPE 20250. 1994.

13. BURGER, J.G. AND SAHUQUET, B.C. Chemical Aspects of In-situ Combustion - Heat of Combustion and Kinetics. Soc. Pet. Eng. J. Trans, Ale, Vol. 253. 1972. Pp 410 - 422.

14. HAYASHITANI, M. Thermal Cracking of Athabasca Bitumen. Ph.D. Thesis, The University of Calgary, Alberta. 1978.

15. H.J. RAMEY. In Situ Combustion WPC-14229. 8th World Petroleum Congress, Moscow, USSR. 1971.

16. A.L. BENHAM, F.H. POETMANN. The thermal recovery process - An analysis of laboratory combustion data. AIME Technical note. 1958.

17. MAMORA D.D. Kinetics of In-Situ Combustion. Ph.D. Thesis. Stanford University. 1993.

18. KAPADIA P.R, KALLOS M.S, , LESKIW C, GATES L.D. Potential for hydrogen generation during in situ combustion of Bitumen. SPE-122028MS. EUROPEC/EAGE. Amsterdam. 2009.

19. L.K. THOMAS, K.H. COATS. Stones Kro methods and modifications. SPE-25289-MS. 1992.

20. LEE D.G, NOURELDIN N.A. Effect of water on the low temperature oxidation of heavy oil. Energy and Fuels 3(6):. DOI: 10.1021/ef00018a009. Pp 713715. 1989.

21. BAGCI S, OKANDAN E. Dry and wet combustion studies of different API gravity crude oils from Turkish oil fields. PETSOC-88-39-58, Annual Technical Meeting, Calgary, Alberta. 1988.

22. SMITH F.W, PERKINS T.K. Experimental and numerical simulation studies of wet combustion recovery process. PETSOC-73-03-05. Journal of Canadian Petroleum Technology, Vol 12. 1973.

23. GARON A.M, WYGAL R.J JR. A Laboratory Investigation of Fire-Water Flooding. SPE 4762. 1974.

24. DIETZ D.N, WEIJDEMA J. Wet and Partially Quenched Combustion. SPE 189. 1968.

Recepción: 15 de enero de 2016

Aceptación: 8 de abril de 2016 